is withheld. A test for early recognition of varicella-zoster virus infection could improve prognosis.

\title{
HOPKINS SYNDROME WITH MYCOPLASMA INFECTION
}

A 3-year-old boy with acute flaccid paralysis of the right lower limb developing one week after treatment and recovery from an acute asthma attack is reported from the Department of Child Health, Milton Keynes General Hospital, UK. Sensation was normal, plantar responses were flexor, deep tendon reflexes were absent in the involved limb, and meningeal signs were negative. MRI of the spine was normal. EMG was consistent with anterior horn cell damage and persisting amyotrophy. Mycoplasma complement fixation test titer and agglutination titer were significantly increased. Erythromycin treatment was without benefit, and paralysis persisted at 12 month follow up. (Acharya $\mathrm{AB}$, Lakhani PK. Hopkins syndrome associated with Mycoplasma infection. Pediatr Neurol Jan 1997;16:54-55). (Response: Dr Lakhani, Department of Child Health, Milton Keynes General Hospital, Eaglestone, Milton Keynes MK6 5LD, UK).

COMMENT. Since the first description of 10 cases of a poliomyelitis-like illness associated with acute asthma in childhood (Hopkins IJ, 1974), 18 additional reports of Hopkins syndrome have been published. Non-polio enteroviruses, varicella and herpes virus type 1 have been implicated in some cases. Mycoplasma is known to exacerbate asthma and has been linked to various neurological disorders, including Guillain-Barre syndrome and transverse myelitis. Amyotrophy and Hopkins syndrome are additional complications of Mycoplasma infection.

\section{CONGENITAL DEVELOPMENTAL DISORDERS}

\section{CONGENITAL FIBROSIS OF EXTRAOCULAR MUSCLES}

The intracranial and orbital pathology and muscle pathology of chromosome 12-linked congenital fibrosis of the extraocular muscles are described in 3 affected members of a family in a report from Children's Hospital, Harvard Medical School, Boston, and other centers. Postmortem examination of 1 and muscle biopsies of 2 patients showed abnormalities in the brain stem, cranial nerves, and extraocular muscles (EOMs): absence of motor neurons of the oculomotor nucleus, loss of axons in III cranial nerve, absence of the superior division of III CN, and atrophic superior rectus and levator palpebrae muscles, showing only a clump of myofibers, connective tissue, and fat. Increased numbers of internal nuclei and central mitochondrial clumping found in other extraocular muscles pointed to an extension of the process beyond the superior division of III $\mathrm{CN}$, and an abnormal development of the EOM lower motor neuron system. (Engle EC, Goumnerov BC, McKeown CA et al. Oculomotor nerve and muscle abnormalities in congenital fibrosis of the extraocular muscles. Ann Neurol March 1997;41:314-325). (Respond: Dr Engle, Division of Genetics, Enders 512, Children's Hospital, 300 Longwood Avenue, Boston, MA 02115).

COMMENT. Congenital fibrosis of extraocular muscles, an autosomal dominant inherited disorder, is characterized by bilateral ptosis and fixation of eyes in a downward and strabismic position. It resembles Brown's syndrome (vertical retraction), and Duane's syndrome (horizontal retraction), associated with fibrosis and aberrant innervation of the rectus lateralis. The pathology 